\title{
Contribution méthodologique pour la validation en milieu réel de nouvelles variétés de plantains
}

\author{
Ludovic TEMPLE $^{a \star}$, Moise KWA ${ }^{b}$, Cyrille EFANDEN ${ }^{b}$, Kodjo TOMEKPE $^{b}$
}

a Cirad-Flhor, TA 50/PS4, bld. de la Lironde, 34398 Montpellier Cedex 5, France

ludovic.temple@cirad.fr

${ }^{b}$ Centre Africain

de Recherches sur Bananiers et Plantains, Carbap, BP 832, Douala, Cameroun

carbap@camnet.cm
* Correspondance et tirés à part

Reçu le 4 novembre 2003 Accepté le 3 mai 2005

Fruits, 2005, vol. 60, p. 163-177 (C) 2005 Cirad/EDP Sciences All rights reserved

DOI: $10.1051 /$ fruits:2005023

RESUMEN ESPAÑoL, p. 177

\section{Methodological contribution for the validation of new plantain varieties in real conditions.}

Abstract - Introduction. Diffusion of new varieties resulting from programs of genetic improvement (hybrid or introductions) sometimes encounters difficulties. To limit this problem, a methodological approach aiming at facilitating the adoption of these varieties by the growers was developed in Cameroon on a plantain-type hybrid. Materials and methods. The approach successively sought to identify on a hierarchical basis, by investigation, the criteria of varietal preferences privileged by the producers; to test, by tests in real conditions, in what ways a new variety answered these criteria; and to evaluate the agronomic performances of the hybrid and a pilot variety according to the diversity of the environments. Results. The results underlined the potential of the approach presented to include/understand the determinants of the adoption of a new variety by the growers. They provide useful information for the piloting of future programs of genetic improvement. They reveal methodological difficulties in the realization of interdisciplinary tests in real conditions, which will have to be taken into account for the improvement of future protocols. Conclusion. The conditions of a new variety adoption vary according to localizations, taking into account the pedoclimatic variables, and according to the growers, taking into account the socio-economic constraints. The varietal offer must thus be diversified to answer the diversity of the requests. The approach taken would gain by being supplemented by investigations of other plantain growers. Our experiment must be regarded as a first contribution to a broader approach.

Cameroon / cooking plantains / varieties / new products / evaluation / innovation adoption

\section{Contribution méthodologique pour la validation en milieu réel de nouvelles variétés de plantains.}

Résumé-Introduction. La diffusion de nouvelles variétés issues de programmes d'amélioration génétique (hybrides ou introductions) rencontre parfois des difficultés. Pour limiter ce problème, une démarche méthodologique visant à faciliter l'adoption de ces variétés par les planteurs a été mise au point au Cameroun sur un hybride de type plantain. Matériel et méthodes. La démarche entreprise a cherché successivement à hiérarchiser, par enquête, les critères de préférences variétales privilégiés par les producteurs ; à tester, par des essais en conditions réelles, en quoi une nouvelle variété répondait à ces critères ; à évaluer les performances agronomiques de l'hybride et d'une variété témoin en fonction de la diversité des milieux. Résultats. Les résultats ont souligné l'intérêt de l'approche présentée pour comprendre les déterminants d'adoption d'une nouvelle variété par les planteurs. Ils fournissent des informations utiles pour le pilotage de futurs programmes d'amélioration génétique. Ils révèlent des difficultés méthodologiques dans la réalisation d'essais interdisciplinaires en conditions réelles, qui devront être pris en compte pour l'amélioration des protocoles futurs. Conclusion. Les conditions d'adoption d'une nouvelle variété varient selon les localisations, compte tenu des variables pédoclimatiques, et selon les planteurs, compte tenu des contraintes socio-économiques. L'offre variétale doit donc être diversifiée pour répondre à la diversité des demandes. La démarche effectuée gagnerait à être complétée par des enquêtes auprès d'autres opérateurs de la filière. Notre expérimentation doit être considérée comme une première contribution à une démarche plus large.

Cameroun / plantain (banane) / variété / produit nouveau / évaluation / adoption de l'innovation 


\section{Introduction}

La recherche agronomique a des difficultés pour accompagner le processus d'innovation variétale lors de la validation de nouvelles variétés hybrides ou introduites ; ces difficultés sont liées à la diversité des contextes socio et agroéconomiques, ainsi qu'à la complexité d'intégration des différentes contraintes ou attentes des opérateurs au sein des filières [1].

Une première stratégie de diffusion mobilise des démarches de sélection participative qui ont été élaborées plutôt sur des cultures annuelles à cycle court. Le producteur y est libre de sélectionner, dans un panel de variétés qui lui sont proposées, celles qui seraient susceptibles de s'adapter au mieux à ses besoins. Pour le bananier, le transfert de ces démarches est cependant difficile. Il suppose que les planteurs puissent comparer les performances des différentes variétés. Or, l'identification variétale sur bananier implique, le plus souvent, de voir le régime. Compte tenu des techniques actuelles de multiplication par rejetonnage en Afrique, il est très rare que les planteurs puissent identifier la variété au moment du prélèvement du rejet car il y a absence de régimes visible. Il s'ensuit un mélange variétal (moyenne de 10 variétés par parcelle) qui rend complexe la possibilité, pour le planteur, de comparer les performances relatives, le cycle de production durant 18 mois et les parcelles étant le plus souvent d'accès difficile.

Une deuxième stratégie, complémentaire de la sélection participative, essaie au préalable de caractériser la demande d'amélioration variétale des producteurs. Elle tente ensuite d'évaluer, dans leurs conditions de production, en quoi une nouvelle variété correspondrait à cette demande et quelles seraient ses performances agronomiques selon la diversité des milieux. Notre contribution méthodologique à l'élaboration d'une telle démarche a mobilisé les acquis d'un dispositif d'enquêtes et d'essais mis en œuvre dans la province du centre Cameroun.

\section{Méthodologie}

La démarche élaborée articule deux étapes successives.
Une première étape hiérarchise, à partir d'une enquête, l'importance relative des critères qui structurent l'adoption d'une nouvelle variété par le producteur.

Une deuxième étape s'appuie sur des essais en milieu réel ; elle teste en quoi les performances d'une nouvelle variété sont conformes à la hiérarchie des préférences établies au préalable. Cette deuxième étape tend à mesurer la performance agronomique de cette nouvelle variété en fonction de la diversité des milieux.

\section{1. Élaboration d'un indicateur d'adoption : I'IPP}

Un indicateur d'adoption a été élaboré qui a été nommé " indice pondéré de préférence variétal " ou IPP.

Les agriculteurs pratiquent depuis des millénaires [2] un processus de sélection variétale qui mobilise un panel de critères variables en fonction des conditions pédoclimatiques et socio-économiques. Dans le cas du plantain en Afrique centrale, les déterminants de cette sélection ont été peu explicités. Pour aider à les révéler, il a été convenu de procéder à la réalisation d'enquêtes et au calcul de l'IPP.

- Les enquêtes ont porté sur 50 producteurs localisés dans la province du centre Cameroun. Elles ont visé à :

- identifier les critères utilisés par ces producteurs pour différencier les variétés,

- hiérarchiser de manière qualitative ces différents critères, du plus important au moins important [3].

- Le calcul de l'IPP est basé sur l'attribution d'un poids " $x$ " au premier critère classé comme prioritaire par les planteurs. La valeur numérique de ce poids est égale au nombre de critères qui ont été identifiés par la réalisation d'enquêtes. Le critère classé en seconde position par cette enquête de départ reçoit un poids " $(x-1)$ " et ainsi de suite de manière récurrente. Il est alors possible de construire un tableau de fréquence des critères, puis de faire la somme des fréquences pondérée. Cela aboutit à l'obtention d'un indice de préférence pour un critère donné. Cet indice de préférence permet alors de réorganiser la hiérarchie initiale des différents critères. 
Le calcul de cet indicateur au seul niveau des producteurs est insuffisant car il ne prend en compte qu'un seul opérateur de la filière. On peut poser cependant pour hypothèse que le producteur, dans la révélation de ses critères de préférence, rend compte partiellement des sollicitations que lui adressent les acheteurs (grossistes, collecteurs, voire consommateurs pour la vente directe). La validation de la démarche impliquerait de reproduire le calcul de l'IPP auprès de chacun des autres opérateurs de la filière, d'analyser ensuite quels sont les opérateurs aptes à jouer un rôle déterminant dans le processus de sélection variétal (l'importance de ce rôle est variable selon les marchés), puis de hiérarchiser l'importance des différents critères en fonction du rôle relatif de ces opérateurs. Cet élargissement méthodologique n'a pu être conduit dans le cadre des travaux présentés. En revanche, un dispositif d'évaluation de la nouvelle variété en fonction de la diversité des conditions de production a été mis en place.

\subsection{Mise en place d'essais d'évaluation en milieu réel}

\subsubsection{Choix des sites et des planteurs}

Deux principales zones d'approvisionnement de Yaoundé en plantain ont été identifiées par des travaux de quantification des flux [4]. Il s'agit des zones de Sa'a et Talba. Par ailleurs, deux zones complémentaires ont été choisies (zone d'Otélé et zone de Sangmélima) pour diversifier les paramètres pédo-climatiques et socio-économiques. Dans chacune des zones sélectionnées, cinq parcelles test ont été plantées (figure 1):

- zone de Sa'a au nord-ouest de Yaoundé : deux parcelles à Nkolmebanga $(4 \mathrm{~km}$ de Sa'a), une à Nkolo 1 (1 km de Nkolmebanga), une à Polo (6 km de Nkolo 1) et une à Nkolélouga (3 km de Polo) ;

- zone de Talba au nord de Yaoundé : trois parcelles à Talba (36 km de Ntui) et deux à Téate (6 km de Talba) ;

- zone d'Otélé au sud-ouest de Yaoundé : cinq parcelles à Mbahguè $(52 \mathrm{~km}$ de Yaoundé) ;
- zone de Sangmélima dans la province du sud : une parcelle à Kondébilong $(36 \mathrm{~km}$ de Sangmélima sur l'axe Yaoundé-Sangmelima), une à Ndjom Yekombo (40 km sur l'axe Sangmelima-Mvomeka'a), une à Nkolotou'outou ( $4 \mathrm{~km}$ avant Ndjom Yekombo), une à Monavebe (2 km à l'ouest de Sangmelima) et une à Bilon $(5 \mathrm{~km}$ de Monavebe).

Deux types de parcelles ont été plantés. Dix-sept parcelles " agriculteur " ont été placées sous la responsabilité d'un planteur ou d'un groupe de planteurs, et trois parcelles "école " ont été confiées à des structures d'encadrement agricole: Centre de développement rural de Talba (CDRT), collège Bullier à Sa'a, école technique agricole (ETA) de Sangmelima.

Les agriculteurs " expérimentateurs " ont été ceux pour qui, dans les zones d'intervention identifiées, le plantain présentait le plus d'importance dans les revenus de l'exploitation [3].

Pendant la durée de notre suivi, quatre parcelles " agriculteur " (parcelle de Nkolo 1 de la zone de Sa'a, de Téate dans la zone de Talba, d'une à Mbahguè de la zone d'Otélé et de celle de Nkolotou'outou de la zone de Sangmelima) et une parcelle " école " (collège Bullier à Nkolmebanga) ont été abandonnées par leurs responsables (décès du planteur ou abandon du foyer). Elles ont donc été éliminées du suivi.

\subsubsection{Matériel mis à disposition des agriculteurs}

Chaque parcelle " agriculteur " a reçu 10 plants hybrides CRBP 39 (hybride plantain de type French) sous la forme de "plants issus de fragments de tiges" ou PIF [5] et 10 plants témoins du cultivar Bâtard (plantain de type Faux-Corne) sous la forme de rejets. Dans les parcelles "école ", 20 hybrides sous forme de PIF et 20 rejets de Bâtards (témoins) ont été plantés. La distribution (tableau I) a été fonction des disponibilités en rejets et en PIF liées aux difficultés de production en station (période de transition des financements du CARBAP).

Chaque responsable de parcelle a reçu des fiches de suivi permettant de noter les dates de floraison, le nombre de feuilles 


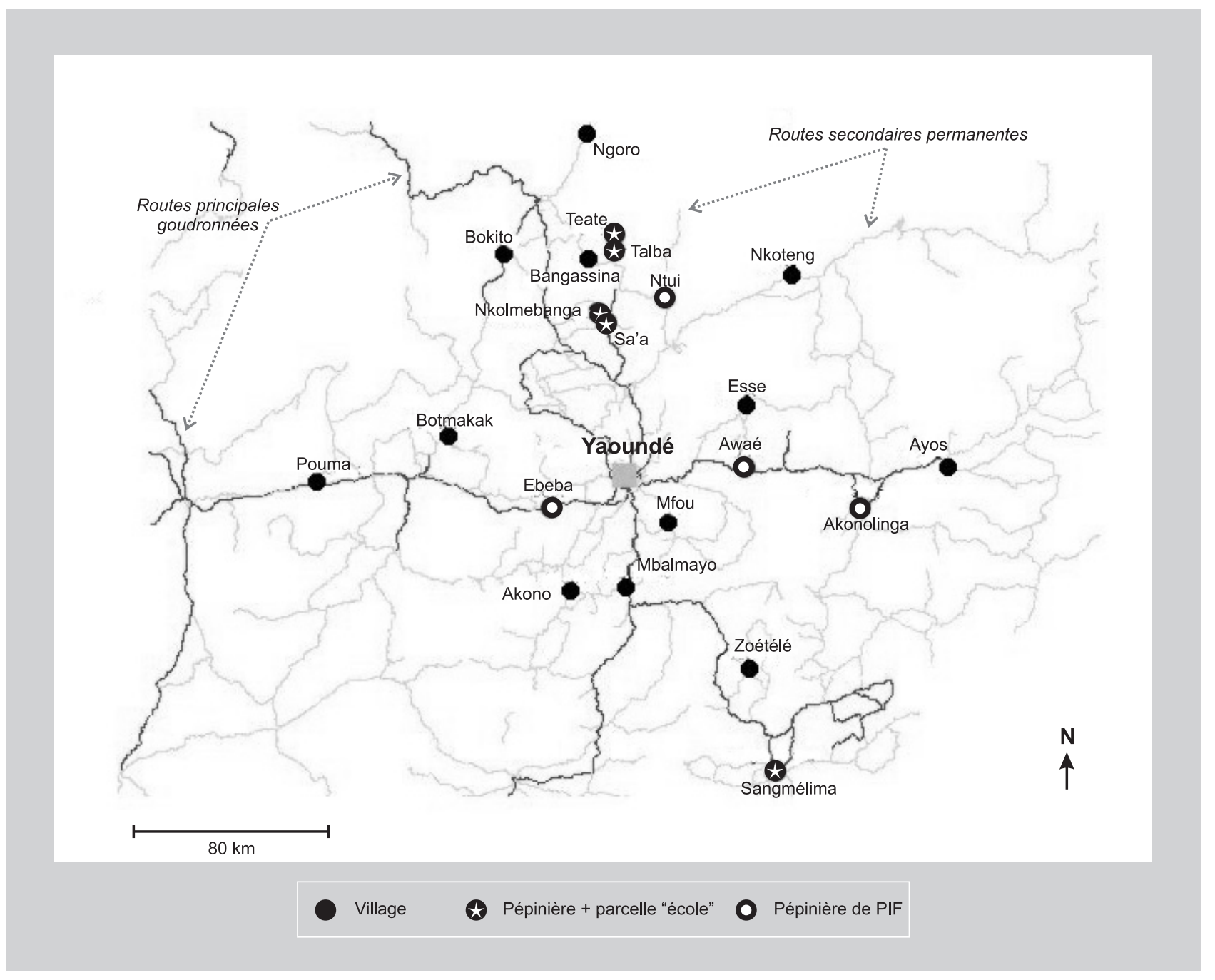

Figure 1.

Localisation des sites de validation du nouvel hybride plantain CRBP 39 en milieu paysan (Centre et Sud Cameroun). vivantes, le poids du régime et les pourcentages de chutes. Les producteurs ont bénéficié d'une formation préalable à l'enregistrement de ces informations et à l'utilisation de pesons.

\subsubsection{Conditions de mise en place des parcelles}

Le protocole d'essai a fait l'objet de négociations entre économistes et agronomes, notamment pour l'établissement de recommandations techniques minimales. Ce protocole avait des d'objectifs d'évaluations agronomiques : comparer la variété hybride au témoin dans des conditions de produc- tion diversifiées ; et des objectifs économiques : tester les déterminants d'adoption d'une nouvelle variété par les producteurs.

Les conditions de mise en place et de plantation ont été gérées par les planteurs, mais des recommandations préalables minimales ont été données avant la plantation. Ces recommandations portant sur le système de culture à adopter et les techniques culturales à appliquer n'impliquaient, a priori, pas de changements majeurs dans les combinaisons de facteurs de production mobilisés habituellement par ces producteurs :

- proscription d'un précédent cultural en plantain, manioc, maïs et arachide l'année 


\begin{tabular}{|c|c|c|c|c|}
\hline \multirow[t]{2}{*}{ Zones } & \multirow[t]{2}{*}{ Localités } & \multirow[t]{2}{*}{ Responsables de la parcelle } & \multicolumn{2}{|c|}{ Quantité de plants distribués } \\
\hline & & & CRBP 39 & Bâtard \\
\hline \multirow[t]{5}{*}{ Talba } & Talba & Afata Réné & 10 & 0 \\
\hline & & Toundé Richard & 10 & 0 \\
\hline & & CDRT & 20 & 20 \\
\hline & Téate & Bessala Guy Bernard & 10 & 0 \\
\hline & & Ngodo Joseph ${ }^{1}$ & 10 & 0 \\
\hline \multirow[t]{5}{*}{ Sa'a } & Nkolmebanga & Assoa Joseph Marie & 10 & 17 \\
\hline & & Collège Bullier ${ }^{1}$ & 20 & 34 \\
\hline & Nkolo I & Njoya Zogo ${ }^{1}$ & 20 & 15 \\
\hline & Polo & Ayissi Ndzana Martin & 10 & 12 \\
\hline & Nkolélouga & Enogo Mbani Martin & 10 & 24 \\
\hline \multirow[t]{5}{*}{ Otélé } & Mbahguè & Mbombog & 10 & 10 \\
\hline & & Mbondo & 10 & 10 \\
\hline & & Biya Laurent ${ }^{1}$ & 10 & 10 \\
\hline & & ONG (Gead) & 10 & 10 \\
\hline & & Mangoun Mathieu & 10 & 10 \\
\hline \multirow[t]{5}{*}{ Sangmelima } & Monavebe & École technique agricole & 30 & 30 \\
\hline & Bilon & Eko Réné & 10 & 10 \\
\hline & Nkolotou'outou & -1 & 10 & 10 \\
\hline & Njom Yekombo & GIC (Pean) & 10 & 10 \\
\hline & Kondébilong & GIC (kulunyulu) & 10 & 10 \\
\hline Total général & & & 250 & 242 \\
\hline
\end{tabular}

qui précédait la mise en place de la parcelle ;

- respect d'un temps de jachères minimal d'au moins 2 ans ;

- proscription des associations culturales susceptibles de générer des concurrences (manioc) ou d'accroître des contraintes parasitaires (maïs, arachide) pendant le cycle de culture ;

- différenciation du bloc des hybrides de celui des témoins pendant la plantation ;
- limitation de la distance de marche à pieds (inférieure à $30 \mathrm{~min}$ ) pour l'accès de l'agriculteur à sa parcelle ;

- réalisation d'une trouaison de $40 \mathrm{~cm} \times$ $40 \mathrm{~cm} \times 40 \mathrm{~cm}$ et d'un écartement de $2 \mathrm{~m} \times$ $3 \mathrm{~m}$;

- bonne manutention des plantules lors de la réception de celles-ci ;

- arrosage des plantules tous les 2 jours pendant au moins 2 semaines après plantation dans le cas d'une absence de pluies ; 
- désherbage régulier ;

- mise de tuteurs lors de la maturation des régimes.

\subsubsection{Le suivi}

En complément des observations réalisées directement par les producteurs, un suivi complémentaire a été confié à un technicien d'appui. Son intervention a commencé 6 mois après les plantations et s'est poursuivie tous les 4 à 2 mois d'intervalle jusqu'à la récolte du régime de premier cycle. Les paramètres suivis dans chaque parcelle ont porté sur les pratiques paysannes, ainsi que sur des indicateurs de croissance de la plante et du rendement. La collecte d'indicateurs de suivi des parcelles a été faite par les producteurs qui avaient été formés au préalable pour cela. Des enquêtes ponctuelles ont été conduites par des assistants de recherche sur des variables techniques : nombre de feuilles vivantes, par exemple. Après les premières récoltes, des enquêtes ont eu lieu pour tester en quoi les résultats obtenus répondaient ou non à la hiérarchie des indicateurs prioritaires de préférences variétales.

\subsubsection{Faiblesses du dispositif}

La disponibilité en témoins ayant été épuisée dans certaines parcelles de Talba, les producteurs se sont vu demander de créer des parcelles témoins avec une variété locale. Dans ces parcelles, la comparaison entre l'hybride et la variété locale n'a pu être réalisée car, compte tenu des techniques de multiplication mobilisées par les producteurs, une forte hétérogénéité est apparue d'une parcelle à l'autre portant sur la variété, l'âge, la qualité sanitaire des rejets sur une partie du dispositif. Cela a conduit à diminuer le nombre d'observations mobilisé dans les analyses statistiques.

Cette validation interdisciplinaire entreprise dans le cadre d'une "recherche action " avait un double objectif : mettre au point d'une démarche méthodologique et produire des informations sur les conditions d'adoption d'un hybride précis. Celle-ci a été confrontée à la difficulté d'adapter des protocoles de validation utilisés en station expérimentale à un milieu réel non contrôlé, où les décisions des planteurs sont soumises à des contraintes spécifiques variables selon les zones. En dépit de ces difficultés, la base de données élaborée apporte des informations utiles sur les déterminants d'adoption de nouvelles variétés de plantains au Cameroun.

\section{Résultats et discussions}

\subsection{Critères de préférence variétale des producteurs}

L'IPP calculé à partir des données issues des enquêtes réalisées a montré que les producteurs privilégient des critères relatifs à la taille du régime et à la grosseur des doigts, puis viennent ceux liés aux qualités organoleptiques, et enfin ceux rendant compte des caractéristiques agronomiques : précocité, pouvoir de rejetonnage, rusticité, résistance aux chutes, durée de vie de la souche (tableau II). Cette hiérarchie des critères est évidemment contextuelle à une filière donnée et une localisation spécifique [6], celle de la zone d'approvisionnement de la ville de Yaoundé.

\subsection{Adoption des protocoles par les producteurs}

\subsubsection{Choix du terrain}

Le choix des sites d'expérimentation a été tributaire des contraintes des planteurs et des structures associées à l'opération. D'une manière générale, les parcelles "école " du CDRT (Talba) et de l'ETA (Sangmelima) ont été localisées sur les aires de pratique agricole de ces structures à vocation éducative. En raison du peu de disponibilité de terrain vierge, les parcelles ont été implantées en bord de marécage ou sur des terres pas toujours optimales pour la culture du bananier. Les parcelles "agriculteur " ont été localisées dans des aires ayant subi des jachères de durées variables, dans d'anciennes plantations de cacaoyers ou sur des terres en fortes pentes. La durée moyenne des jachères pour l'ensemble de ces sites était de 8 ans.

\subsubsection{Précédents culturaux}

À Sa'a, toutes les parcelles suivies étaient établies sur de vieilles jachères jadis cultivées en vivriers. À Talba, $75 \%$ des parcelles 
étaient sur des jachères vivrières et $25 \%$ sur des anciennes cacaoyères. A Sangmelima, les précédents culturaux des 3 dernières années ont été : arachides et maïs, pépinière maraîchage à l'ETA, palmier à huile à Otélé.

\subsubsection{Pratique du brûlis, trouaison et arrosage}

À Otélé et Sangmelima, aucune parcelle n'avait subi de brûlis ; en revanche, l'une des cinq parcelles de Sa'a avait été partiellement brûlée, et à Talba (zone pionnière), en dépit des recommandations, un brûlis avait été pratiqué sur $75 \%$ des parcelles. Les recommandations ont été le plus respectées dans les zones présentant le plus de contraintes pour l'accès au foncier.

Les normes conseillées pour la trouaison ont été respectées sur les 3/4 des parcelles de Sangmelima, sur la moitié de celles de Talba, sur $1 / 3$ de celles de Sa'a et sur 1/4 des parcelles d'Otélé.

Pour l'ensemble du dispositif, l'arrosage en début de cycle n'a été pratiqué que sur quatre parcelles malgré la sévérité de la saison sèche et les recommandations établies. En l'occurrence, la disponibilité de points d'eau et l'absence de moyens pour le transport de l'eau ont été des facteurs limitants importants.

\subsubsection{Associations culturales}

Dans toutes les parcelles de Talba, les planteurs ont introduit de jeunes cacaoyers 6 à 8 mois après la mise en place du dispositif, et parfois quelques pieds de macabo. À Otélé, une association avec du palmier à huile a pu être notée. Les autres parcelles du dispositif ont été conduites en monoculture durant le premier cycle.

\subsubsection{Pratique du désherbage}

La pratique du désherbage n'a pas été la même selon qu'il s'agissait de parcelles " école " ou " agriculteur ". Ainsi, le désherbage a été effectué régulièrement dans les parcelles " école " et il a été plus sporadique dans les parcelles "agriculteur". En fait, les parcelles "école " étant sous la responsabilité de structures éducatives, l'entretien des plantations de plantain a fait partie des

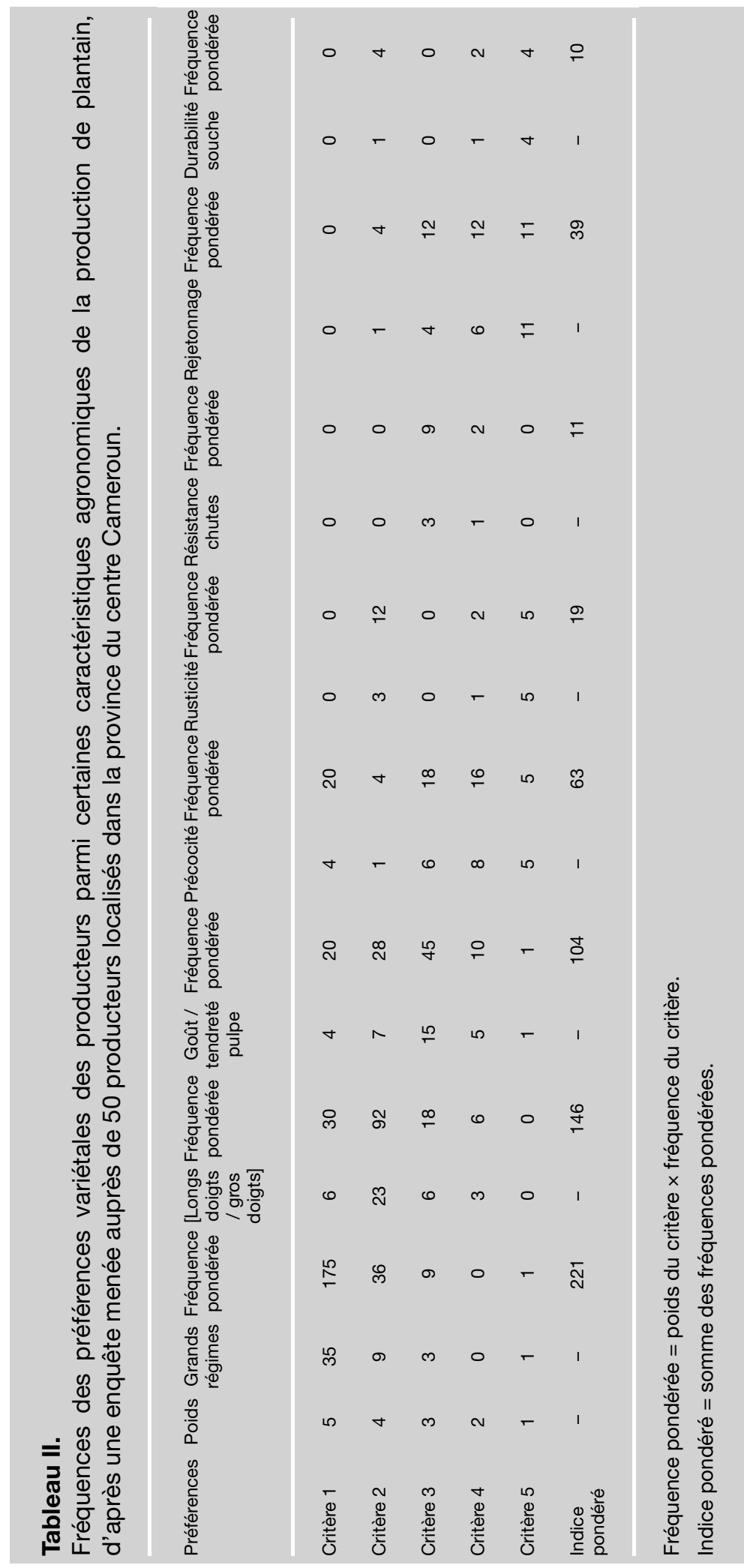


programmes de travaux pratiques. Le désherbage a donc été régulièrement effectué par les élèves des diverses institutions concernées.

En revanche, dans les parcelles " agriculteur ", le désherbage n'a eu généralement lieu que lors du passage de la personne chargée du suivi. Les planteurs ayant respecté la recommandation minimale de ne pas associer le maïs au plantain, ont supprimé le désherbage qui se faisait dans le cadre de cette association. Ainsi, la plupart des parcelles testant l'adoption de l'innovation variétale se sont trouvées moins bien entretenues que les parcelles de variétés locales cultivées en associations culturales. Cet exemple révèle une incompatibilité des recommandations de la recherche, qui avaient pourtant été considérées comme minimales, et des pratiques des agriculteurs. Il était demandé de ne pas associer du maîs à la plantation des plantains et d'assurer un désherbage régulier, alors que les planteurs ont comme objectifs de rechercher des économies de gamme en optimisant le temps de travail du désherbage sur des associations culturales

\subsubsection{La fertilisation}

En dehors de l'urée appliquée lors de la plantation à la dose de $15 \mathrm{~g}$ par pied dans la zone de Sangmelima, seule la parcelle d'Otélé a reçu un apport minime d'engrais sous forme de formulation NPK. Des apports en matières organiques (herbes mortes, purin de porc) ont été observés dans la parcelle de Sangmelima.

\subsubsection{Autres pratiques}

Bien qu'il n'ait pas été fait de façon systématique dans les différentes parcelles, l'effeuillage a été pratiqué dans toutes les zones sans que le nombre moyen de pieds effeuillés par parcelle soit toutefois connu. Les responsables de parcelles n'ont ni marqué les pieds sur lesquels cette pratique avait été réalisée, ni noté le nombre total de feuilles coupées par pied. L'œilletonnage n'a pratiquement pas été réalisé.

Le protocole, qui s'est révélé différemment appliqué d'un planteur à l'autre, illustre la difficulté d'évaluer, en milieu réel, une nouvelle variété en utilisant des critères d'appréciation établis en station de recherche. Malgré le caractère peu contraignant a priori des recommandations établies qui n'exigeaient pas l'utilisation d'intrants, leur mise en ouvre a été hétérogène du fait de contraintes localisées et d'objectifs divergents entre les planteurs et les chercheurs.

\section{3. Évaluation d'une nouvelle variété par les planteurs}

Le calcul de l'indice de préférence (IPP) réalisé dans la première étape d'enquête a permis de hiérarchiser les variables privilégiées par les planteurs pour la sélection d'une variété, dans une zone donnée (tableau II). Cette hiérarchie a ensuite été utilisée pour interroger les planteurs sur leur appréciation de la nouvelle variété par rapport à une variété témoin locale.

- Pour la taille du régime (critère 1), 70 \% des producteurs ont déclaré obtenir de meilleurs résultats avec la nouvelle variété hybride CRBP 39 qu'avec la (les) variété(s) témoin(s) (figure 2).

- Le deuxième indicateur de préférence variétale révélé par le calcul de l'IPP a été la grosseur des doigts. Ce critère est également classé comme prioritaire en tant que variables qualitatives par les consommateurs des villes [7]. Près de $60 \%$ des planteurs considèrent que l'hybride proposé obtient de meilleurs résultats que la variété témoin.

- En troisième position, les producteurs ont identifié le critère du goût comme indicateur de préférence variétale. Pour $55 \%$ d'entre eux la nouvelle variété présente un avantage comparatif (figure 3). Les bons résultats relatifs de l'hybride à partir de ce troisième critère sont cependant en diminution par rapport aux évaluations touchant les critères précédents. Par ailleurs, le pourcentage de personnes (36\%) évaluant l'hybride "moins bon " que la variété témoin atteint, dans le cas de ce critère, le plus haut niveau par rapport aux résultats obtenus avec les quatre autres critères définis. Cela s'explique par le fait que, si au stade vert le goût et la "tendreté " (pulpe ferme) de l'hybride sont meilleurs, cette appréciation s'inverse 
au stade mûr ; l'hybride a alors en effet une consistance beaucoup "trop molle " qui, à ce stade, conduirait à lui attribuer des propriétés culinaires se rapprochant plus de la banane que du plantain. Les retours des acheteurs (grossistes) enregistrés auprès des producteurs révèlent par ailleurs que la rapidité de mûrissage de cet hybride par rapport aux variétés locales pose un problème de commercialisation en cas de méventes. Cette observation confirme celle des sélectionneurs qui ont pu constater aussi que, après la récolte, cet hybride mûrissait plus vite que les variétés locales. Elle soulève deux interrogations sur l'adoption future de cette nouvelle variété :

- elle implique que le délai entre la coupe du régime et sa mise à disposition au consommateur soit plus court que celui utilisé pour les variétés locales. De fait, cette nouvelle variété pourrait avoir du mal à être adoptée de manière significative dans les zones éloignées des marchés, ou peu accessibles ;

- le caractère "trop mou " de l'hybride permet difficilement de le consommer pilé, or cette forme de préparation est dominante chez certains groupes ethnolinguistiques et dans certaines régions d'Afrique centrale.

- Le quatrième critère (IPP de 63) est la précocité, c'est-à-dire la longueur du $1^{\mathrm{er}}$ cycle de production. Par rapport à ce critère 4 , $73 \%$ des agriculteurs interrogés considèrent que l'hybride a un cycle plus court que les variétés témoins testées (figure 4).

- Le cinquième critère est le pouvoir de rejetonnage d'une variété. Avec ce critère, l'hybride CRBP 39 a obtenu son moins bon résultat : $54 \%$ des producteurs ont déclaré que les variétés témoins étaient plus performantes que l'hybride quant au nombre de rejets produit. Ce résultat est en partie confirmé par le fait qu'en station beaucoup de plantains produisent plus de rejets que ne le fait l'hybride. Cependant le degré d'inhibition plus ou moins grand du rejet successeur n'a pas été pris en considération, or si le CRBP 39 produit un moins grand nombre de rejets, ceux-ci sont connus pour être moins inhibés que les rejets issus des cultivars témoins. Cela étant, il a pu être noté qu'il était plus difficiles d'évaluer avec rigueur ce

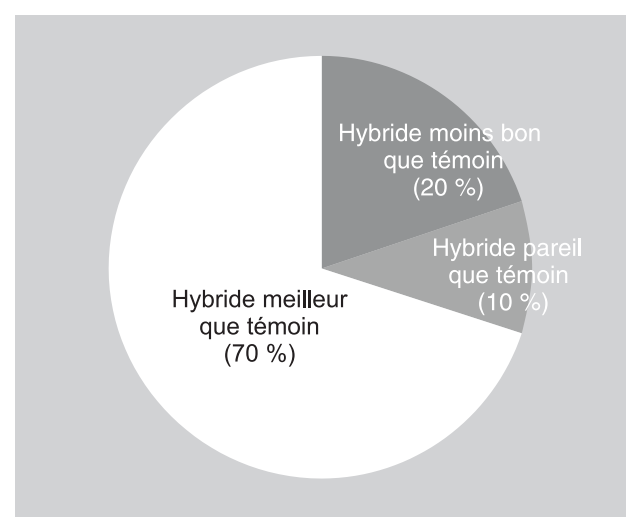

Figure 2.

Appréciation du nouvel hybride plantain CRBP 39 par les producteurs à partir du critère de taille du régime.

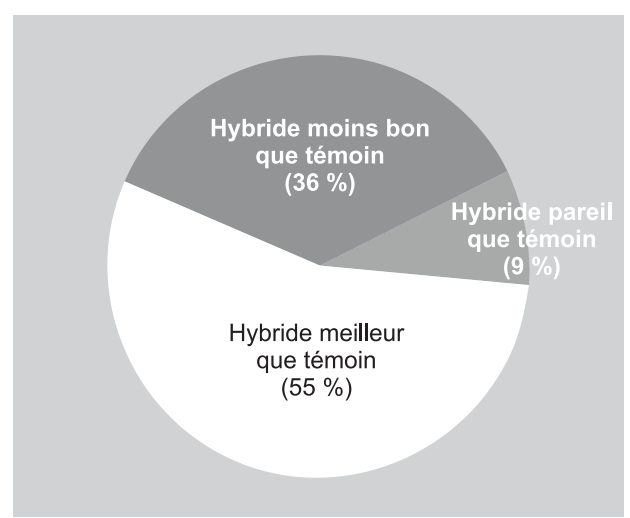

Figure 3.

Appréciation du nouvel hybride plantain CRBP 39 par les producteurs à partir du critère de goût.

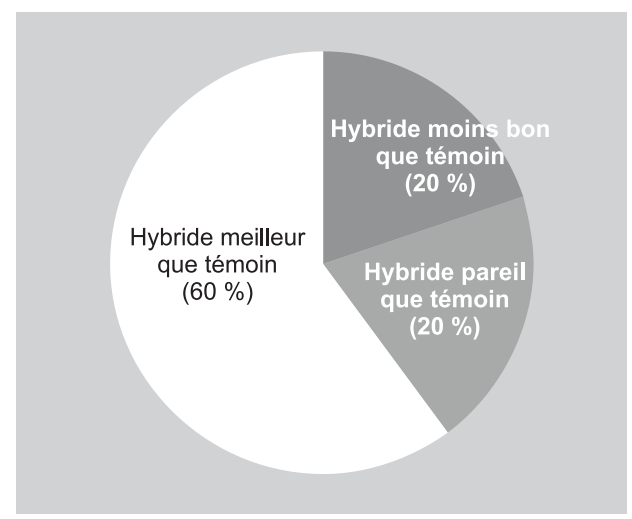

Figure 4.

Appréciation du nouvel hybride plantain CRBP 39 par les producteurs à partir du critère de durée du premier cycle. caractère de production de rejets en milieu réel qu'en station de recherche. En effet l'existence d'une nouvelle variété chez les producteurs expérimentateurs a entraîné de nombreux vols de rejets dans les parcelles d'hybrides, ce qui a plus ou moins faussé les comptages. 


\begin{tabular}{|c|c|c|c|c|c|c|}
\hline Variété & $\begin{array}{l}\text { Poids moyen } \\
\text { du régime } \\
(\mathrm{kg})\end{array}$ & Variance & Écart-type & $\begin{array}{l}\text { Pourcentage } \\
\text { de chute }\end{array}$ & $\begin{array}{l}\text { Pourcentage } \\
\text { de récolte }\end{array}$ & $\begin{array}{c}\text { Rendements pour } \\
1666 \text { pieds } \\
\left(\mathrm{t} \cdot \mathrm{ha}^{-1}\right)\end{array}$ \\
\hline $\begin{array}{l}\text { Hybride } \\
\text { CRBP } 39\end{array}$ & 15,7 & 39,4 & 6,3 & 51,9 & 48,1 & 12,8 \\
\hline $\begin{array}{l}\text { Bâtard } \\
\text { (témoin) }\end{array}$ & 13,4 & 41,3 & 6,4 & 66,3 & 33,7 & 9,0 \\
\hline
\end{tabular}

En pondérant les résultats obtenus pour chacun des critères par leur IPP relatif et en faisant la somme des résultats sur chacun des critères, il a été possible d'obtenir un indicateur synthétique permettant de comparer l'appréciation de l'hybride et celle de la variété témoin. Selon cet indicateur, $60 \%$ des producteurs préfèreraient l'hybride au témoin et $23 \%$ opteraient plus pour le témoin que pour l'hybride.

L'approche présentée permet de donner une première évaluation par les planteurs d'une nouvelle variété et, par conséquent, des déterminants de son adoption en fonction de conditions localisées. Si cette démarche implique d'être complétée par une intégration des opérateurs de l'aval pour une réelle validation, elle produit, par le dispositif de terrain mis en place, des informations sur les résultats agronomiques obtenus en conditions réelles de production.

\subsection{Difficultés d'analyse des observations agronomiques en milieu réel}

Le suivi des paramètres agronomiques en milieu réel chez les agriculteurs n'a pas été aussi efficace qu'en station. Ainsi le rapport [somme des régimes récoltés ou perdus / somme des pieds plantés], appelé pourcentage d'observations, a été de $89 \%$ pour l'hybride et de $55 \%$, pour le témoin. Cela signifierait que les producteurs ont montré moins d'intérêt pour s'impliquer dans le suivi d'une variété considérée comme connue (variété Bâtard) que pour évaluer une nouvelle variété (CRBP 39). Par ailleurs, sur le dispositif de suivi mis en place, les taux de chute avant récolte ont été extrêmement importants ( $52 \%$ pour l'hybride et $66 \%$ pour le témoin, tableau III). Deux explications peuvent être proposées : d'une part, les plantations des parcelles ont eu lieu entre juin et juillet, soit après les dates considérées comme optimales dans la zone de suivi, compte tenu de conditions de production sans irrigation ; d'autre part, l'année d'observation a été caractérisée par une saison sèche importante. Les conditions agronomiques et climatiques des parcelles étudiées ont donc été relativement défavorables à une bonne production. Malgré ces difficultés, l'exploitation de la base de données constituée a permis de comparer le pourcentage de récolte (variable centrale du rendement) entre les deux variétés hybride et témoin, en condition réelle de production (sans intrants). Cette comparaison a été réalisée par l'utilisation d'analyses statistique classiques de comparaison bilatérale de pourcentage, à partir de méthodes paramétriques au seuil de $5 \%$; elles ont conduit au calcul d'un rapport critique (RC). Ce rapport de 2,3 conduit à accepter l'hypothèse selon laquelle les différences de pourcentage de récolte sont statistiquement différentes entre l'hybride et le témoin. Ce résultat confirme l'appréciation qualitative obtenue par enquête auprès des producteurs à propos des caractéristiques agronomiques de l'hybride trouvés meilleures que celles de la variété témoin (tableau III).

Ces bons résultats soulèvent cependant une interrogation. En effet, l'hybride a pour principale caractéristique d'être résistant à la 


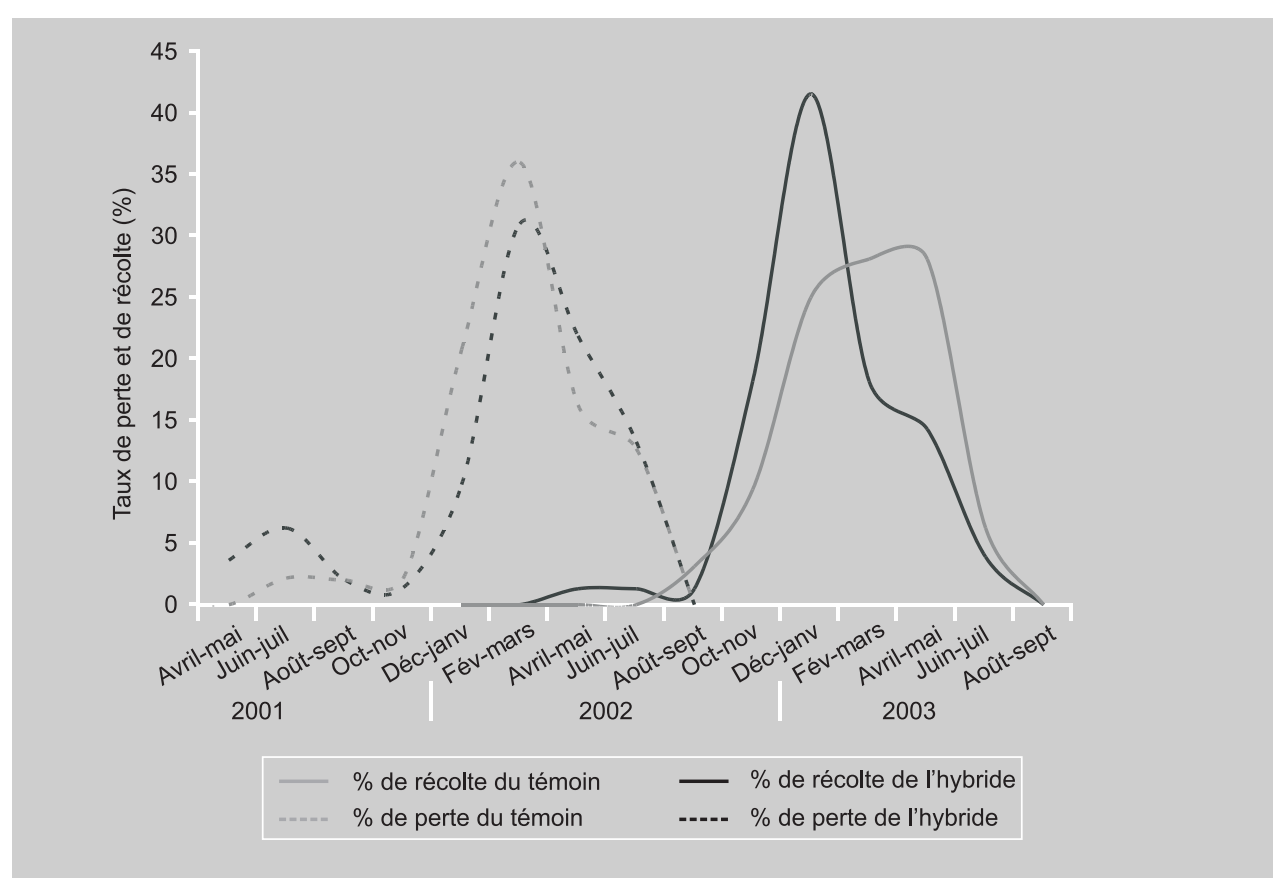

maladie des raies noires [8], or la différence la plus significative entre les performances de CRBP 39 et celles de la variété témoin a porté sur le pourcentage de chute des plants à la floraison, plus important pour le témoin que pour l'hybride. A priori, cette chute est déterminée par les contraintes parasitaires. S'il existe certes une relation entre un meilleur état sanitaire des feuilles et le bon état sanitaire du système racinaire [9], cette relation est établie dans des conditions de forte pression de la maladie des raies noires. Or ces conditions ne correspondent pas tout à fait à celles de la zone dans laquelle la production de l'hybride a été comparée à celle du témoin [10].

\subsubsection{Impact du matériel végétal de plantation}

La différence du pourcentage de chute observé entre les plantains hybrides et les plants témoins pourrait être liée au matériel végétal utilisé pour la mise en place des parcelles : les hybrides ont été fournis sous forme de plantules issues de micropropagation [5] alors que la variété témoin a été livrée sous forme de rejets classiques. Cependant, ces rejets étant parés et prélevés sur une parcelle traitée, la qualité sanitaire les deux matériels a donc été a priori de qualité comparable vis-à-vis des nématodes et charançons. La différence entre ces deux matériels pourrait donc se situer au niveau de leur propriété de reprise, liée à l'effet de rajeunissement plus important pour les plantules issues de PIFs que pour les rejets classiques.

Une analyse de la répartition des pertes et des récoltes au cours de l'année (figure 5) a permis certaines remarques :

- les pertes par chute ont eu lieu à la même période pour les deux variétés testées : elles ont été maximales entre les mois de décembre et mars qui correspondent à la saison sèche et à la floraison des plantains dans la province du Centre Cameroun ;

- la période de récolte de l'hybride a été avancée par rapport à celle du témoin, ce qui confirme les appréciations qualitatives des producteurs qui ont mis en évidence la précocité de l'hybride par rapport à la variété témoin.

\section{Figure 5.}

Évolution des pertes (\%) comparées entre le nouvel hybride plantain CRBP 39 et le plantain témoin (cultivar Bâtard) au cours d'un cycle de production et récoltes obtenues (centre du Cameroun). 


\subsubsection{Impact des autres variables}

Le nombre moyen de feuilles vivantes à la floraison de 10,7 pour l'hybride n'a pas été significativement différent de la moyenne de 10,3 obtenue pour le témoin. Ainsi, si on retient pour indicateur de cette résistance ce paramètre de nombre moyen de feuilles vivantes à la floraison, la résistance à la maladie des raies noires de l'hybride, qui est sa caractéristique principale, n'expliquerait peut être pas directement la différence de rendement observée. En effet, dans la mesure où l'impact de la maladie des raies noires se fait surtout sentir entre la floraison et la récolte, il aurait fallu comparer également, entre l'hybride et le témoin, le nombre de feuilles vivantes à la récolte apte à donner une indication sur le remplissage des fruits pendant la maturation au champ. Les données collectées n'ont pas permis de disposer de cette information. Cette hypothèse conduirait à envisager une amélioration du protocole d'observation dans le futur.

\subsubsection{Impact de la zone}

L'objectif méthodologique du dispositif a également été de tester diverses situations du point de vue de différents paramètres (pédo-climatiques, socio-économiques) sans prétendre rendre compte de la diversité existante. Les données mobilisables ont permis de tester, à partir des techniques des tests paramétriques conventionnelles, la relation entre les zones de production (Talba et Sa'a) et la différence de pourcentage de récolte entre l'hybride CRBP 39 et la variété témoin :

- une première comparaison a testé le caractère significatif de la différence de pourcentage de récolte entre l'hybride et le témoin, dans chacune des deux zones prises séparément. La différence de pourcentage de récolte a été significative dans la zone de Talba (rapport critique $\mathrm{RC}=2,38$ ), mais elle ne l'a pas été dans la zone de Sa'a $(\mathrm{RC}=0,67)$;

- une deuxième comparaison a mis en évidence que la différence de pourcentage de récolte entre les deux zones n'était pas significative $(\mathrm{RC}=1,82)$.

Ces observations, en partie contradictoires, ne sont pas conformes aux attentes et ne permettent pas de conclusions définitives. Elles révèlent que les résultats comparatifs entre deux variétés sont susceptibles de varier en fonction de la spécificité des milieux localisés et que, par conséquent, les bons résultats d'un hybride par rapport à une variété témoin pourraient être amplifiés, ou ne pas se vérifier, dans des zones aux conditions agronomiques et techniques différentes. La prise en compte du poids de régime comme deuxième variable explicative du rendement aurait permis de compléter l'analyse. Or, les données mobilisables sur les poids des régimes n'ont pas permis de réaliser des tests statistiques pour vérifier si cette différence était significative à partir des méthodes paramétriques utilisées car le poids moyen du régime a été calculé sur un pourcentage d'observation de l'échantillon de $77 \%$ pour l'hybride, mais seulement de $15 \%$ (nombre d'observations inférieur à 30) pour le témoin. Malgré la distribution de balances aux planteurs, ceux-ci ont rapidement négligé de peser les régimes. Cela a constitué une limite dans la capacité à évaluer, en milieu réel, des observations agronomiques. Poursuivant l'analyse des données obtenues, d'autres variables explicatives de la différence de pourcentage de récolte entre l'hybride et le témoin ont été recherchées parmi les pratiques culturales jusqu'ici considérées comme homogènes.

\subsubsection{Impact du précédent cultural}

L'échantillon de 15 parcelles, réparties en 5 types de systèmes de culture fonctions du précédent cultural, n'a pas permis non plus d'effectuer des tests statistiques sur l'impact du précédent cultural sur des variables du rendement. Cependant, le calcul des pourcentages de récolte par type de parcelles a mis en évidence de très mauvais résultats dans les parcelles à précédent maïs (tableau IV). Cela est en conformité avec les résultats des recherches effectuées en station qui déconseillent la rotation plantains / maïs du fait des problèmes conjoints de nématodes entre certaines variétés de maïs et le bananier. Les parcelles en précèdent maraîchage (amarantes principalement) ont présenté les taux de récolte les plus élevés (80\%), ainsi que les parcelles à précédent forestier (vierges). 


\begin{tabular}{|c|c|c|c|c|c|c|c|}
\hline \multirow{2}{*}{$\begin{array}{l}\text { Précédent } \\
\text { cultural }\left({ }^{1}\right)\end{array}$} & \multicolumn{3}{|c|}{ Hybride CRBP 39} & \multicolumn{3}{|c|}{ Témoin Bâtard } & \multirow{2}{*}{$\begin{array}{l}\text { \% de régimes } \\
\text { récoltés } \\
\text { (moyenne) }\end{array}$} \\
\hline & Récolté & Total & $\%$ récolté & Récolté & Total & $\%$ récolté & \\
\hline Jachère (1) & 1 & 10 & 10 & 3 & 10 & 30 & 20 \\
\hline Maïs (4) & 11 & 39 & 28 & 7 & 29 & 24 & 26 \\
\hline Pérenne (2) & 12 & 20 & 60 & 0 & 6 & 0 & 30 \\
\hline Vierge (6) & 33 & 68 & 49 & 14 & 39 & 36 & 42 \\
\hline Maraîcher (2) & 20 & 23 & 87 & 8 & 11 & 73 & 80 \\
\hline Total & 77 & 160 & - & 32 & 95 & - & - \\
\hline
\end{tabular}

\section{Conclusion}

Trois résultats majeurs ont pu être obtenus grâce à la méthodologie utilisée. Tout d'abord, la démarche adoptée pour l'élaboration d'un indicateur de préférence variétale est reproductible. Elle produit par ailleurs des informations utiles sur l'appréciation des conditions d'adoption d'une nouvelle variété dans une zone donnée. Elle peut ainsi permettre, avant la diffusion d'une nouvelle variété, de s'assurer que les conditions d'accueil lui sont favorables et de disposer, le cas échéant, d'éléments judicieux pour choisir la variété qui aurait la plus forte probabilité de réussir. Cela diminue le risque d'échec pris par les planteurs et renforce a priori l'impact des résultats de la recherche sur la diffusion de ses introductions variétales. Sur un autre plan, la démarche présentée permet de hiérarchiser les critères les plus importants pour l'adoption d'une nouvelle variété ; ils peuvent alors être mieux pris en compte pour sélectionner les hybrides placés en phase de validation chez les producteurs. Comme déjà évoqué, cette démarche gagnerait à être complétée par des enquêtes auprès d'autres opérateurs de la filière. L'expérimentation que nous avons effectuée est une première contribution à une démarche plus large.

Les difficultés méthodologiques qui ont accompagné la réalisation des essais de vali- dation en milieu réel sur le bananier plantain suggèrent un certain nombre de réflexions qui pourront être utiles pour l'amélioration des protocoles. En premier lieu, elles conduisent à recommander aux chercheurs de contrôler eux-mêmes les observations agronomiques qui les intéressent au lieu de les confier aux producteurs. En effet, ceux-ci se sont montrés difficilement mobilisables pour suivre des paramètres qui ne leur étaient pas directement utiles pour conduire leurs plantations. En deuxième lieu, les problèmes rencontrés conduisent à conseiller d'éviter de mettre au point des protocoles interdisciplinaires d'évaluation en milieu réel, chaque discipline ayant ses interrogations spécifiques. Ainsi, si l'agronome a besoin d'homogénéiser les variables techniques pour établir une liaison statistique fiable entre une variété, un contexte pédoclimatiques et un rendement, le socio-économiste a besoin, pour comprendre les déterminants de l'adoption d'une nouvelle variété, de respecter la liberté de choix des planteurs dans les itinéraires techniques mis en œuvre. Viser ces deux objectifs dans un même protocole d'essai contraint à envisager des compromis méthodologiques qui peuvent se traduire par l'obtention de résultats incomplets pour les deux disciplines.

La méthode que nous avons testée pour la validation en milieu réel de nouvelles 
variétés de plantains a permis finalement de produire quelques observations intéressantes à synthétiser.

- Les conditions d'adoption d'une nouvelle variété, donc, a priori, son impact futur, varient selon les localisations, compte tenu des variables pédoclimatiques, et selon les planteurs, compte tenu des contraintes socio-économiques. L'offre variétale doit donc être diverse pour répondre à cette diversité de demandes.

- Les propriétés post-récoltes de nouvelles variétés en termes de durée de mûrissement ou de dureté de la pulpe sont susceptibles de limiter l'adoption d'une variété dans certaines zones du fait de pratiques alimentaires particulières et de certaines contraintes logistiques de commercialisation.

- Les augmentations de rendement liées à l'utilisation d'une nouvelle variété et/ou l'utilisation d'un matériel de plantation nouveau confirment qu'il serait possible d'améliorer les performances de l'agriculture vivrière par une démarche d'innovation variétale qui ne nécessiterait pas d'intensifier les systèmes de production en intrants.

Ces conclusions incitent les acteurs de la recherche agronomique à s'impliquer d'avantage dans l'accompagnement et la diffusion de ses produits ; l'enjeu en est une efficacité accrue en terme d'impact sur le développement et en capacité à identifier le besoin de structures relais de type " centres techniques ", susceptibles de faciliter la validation des nouvelles variétés. Les informations obtenues par nos travaux permettent d'approfondir la connaissance des besoins à satisfaire. Elles sont donc mobilisables comme éléments d'orientations possibles pour la mise en œuvre de programmes d'amélioration génétique.

\section{Références}

[1] Hocdé H., Lançon J., Trouche G., La sélection participative : impliquer les utilisateurs dans l'amélioration des plantes, Actes Montpellier sept. 2001, Cirad, Montpellier, France, 2001, $148 \mathrm{p}$.

[2] Ipotesi E., Quels sont les facteurs d'implication des utilisateurs dans les programmes de sélection? Rapp. ESAP/CIRAD, Purpan, Toulouse, France, 2002, $30 \mathrm{p}$.

[3] Efanden C., Temple L., Tomekpe K., Sélection variétale par des producteurs du centre du Cameroun, Infomusa 12 (1) (2003) 4-8.

[4] Foudjem D., Temple L., Quantification of the flows of plantains in Yaoundé, Rapp. Fond de Recherche sur Base compétitive, CARBAP, Yaoundé, Cameroun, 2003, 9 p.

[5] Kwa M., Activation de bourgeons latents et utilisation de fragments de tige du bananier pour la propagation de masse de plants en conditions horticoles in vivo, Fruits 58 (6) (2003) 315-328.

[6] Pierrot J., Achard R., Temple L., Abadie C., Fogain R., Déterminants de la production de plantain dans le sud-ouest du Cameroun: intérêt d'un observatoire, Fruits 57 (2) (2002) 75-86.

[7] Dury S., Bricas N., Tchango J., Temple L., Bikoï A., The determinant of urban plantain consumption in Cameroon, Food Qual. Prefer. 32 (2002) 81-88.

[8] Cohan J.P., Abadie C., Tomekpe K., Tchango Tchango J., Performances agronomiques et résistance à la maladie des raies noires de I'hybride CRBP-39, Infomusa 12 (1) (2003) 29-32.

[9] Blomme G., Swennen R., Tenkuano A., Ortiz R., Vuylsteke D., Estimation du développement des racines à partir des caractéristiques des parties aériennes chez les bananiers et les bananiers plantains, Infomusa 10 (1) (2001) 15-17.

[10] Temple L., Foaguegue A., Les contraintes de la production de plantain dans la province du centre Cameroun, Infoplantain 52 (2002) 5-7. 


\section{Contribución metodológica para la validación de nuevas variedades de plátanos en un entorno real.}

Resumen - Introducción. La difusión de nuevas variedades resultantes de programas de mejora genética (híbridos o introducciones) resulta ser a veces dificultosa. Para limitar este problema, se formalizó un procedimiento metodológico con un híbrido del tipo plátano, destinado a facilitar la adopción de estas variedades por parte de los cultivadores en Camerún. Material y métodos. El procedimiento realizado pretendió sucesivamente, en un primer lugar, jerarquizar mediante investigación los criterios de preferencias de las variedades privilegiadas por parte de los productores; en segundo lugar, a testar mediante pruebas en condiciones reales los aspectos con los que una nueva variedad respondía a estos criterios; en tercer lugar, a evaluar los resultados agronómicos del híbrido y los de una variedad testigo en función de la diversidad del entorno. Resultados. Los resultados subrayaron el interés del acercamiento presentado para comprender cuáles son los determinantes para la adopción de una nueva variedad por parte de los cultivadores. Proporcionan, además, informaciones útiles para guiar futuros programas de mejora genética. Asimismo revelan dificultades metodológicas en la realización de pruebas interdisciplinarias en condiciones reales, que deberán tenerse en cuenta para mejorar los protocolos futuros. Conclusión. Las condiciones de adopción de una nueva variedad varían según la localización, considerando las variables pedoclimáticas, y según los mismos cultivadores, considerando las dificultades socioeconómicas. La oferta de variedades debe pues diversificarse para cubrir la diversidad de las demandas. El planteamiento efectuado mejoraría de ser completado por más investigaciones realizadas por otros operadores del sector. Nuestro experimento debe considerarse como una primera contribución a un procedimiento más amplio.

Camerún / plátano de cocinar / variedades / productos nuevos / evaluación / adopción de innovaciones

To access this journal online: www.edpsciences.org 